Somnologie $2016 \cdot 20: 4$

DOI 10.1007/s11818-016-0045-9

Online publiziert: 2. Februar 2016

(c) Springer-Verlag Berlin Heidelberg 2016

\title{
Online-Einreichung von Manuskripten für die „Somnologie“
}

Weitere Informationen zum Verfassen und Einreichen von Manuskripten finden Sie in der nebenstehenden Infobox.

Ihre

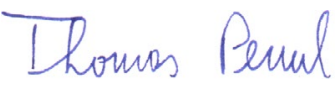

Prof. Dr. Thomas Penzel

Schriftleitung Somnologie

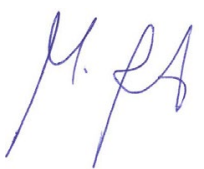

Prof. Dr. Michael Arzt

Schriftleitung Somnologie

\section{Schriftleitung}

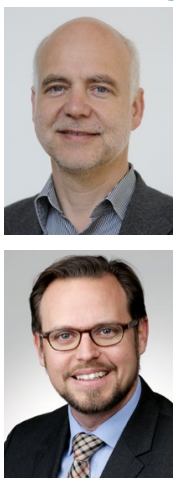

Prof. Dr. Thomas Penzel Charité Campus Mitte Schlafmedizinisches Zentrum Luisenstr.13 10117 Berlin somnologie@charite.de ein stabiles Dokument, das überall an jedem Computer gleich aussieht.

- Sie beschleunigt den Begutachtungsprozess: Das Redaktionssystem ermöglicht die nahtlose Verbindung zwischen Einreichung, Begutachtung, eventueller Revision und schließlich Veröffentlichung.

- Ihre Daten sind sicher: Sie werden auf dem sicheren zentralen Datenserver gespeichert, der regelmäßig gesichert wird, sodass ein Datenverlust nicht vorkommt. Innere Medizin II,

\section{Prof. Dr. Michael Arzt}

Schlafmedizinisches Zentrum der Klinik und Poliklinik für Pneumologie, Universitätsklinikum Regensburg Franz-Josef-Strauß-Allee 11 93053 Regensburg Michael.Arzt@klinik. uni-regensburg.de

Assistenz der Schriftleitung

Wioleta Gorgolik

Charité Campus Mitte

Schlafmedizinisches Zentrum

Luisenstr.13

10117 Berlin

somnologie@charite.de

\section{Infobox}

Manuskript verfassen

- Bitte folgen Sie auf der Homepage www.somnologie.springer.de dem Link „Für Autoren“.

- Hier finden Sie die Autorenleitfäden und Musterbeiträge mit Hinweisen für die Manuskriptgestaltung.

\section{Manuskript einreichen}

- Bitte klicken Sie auf „Manuskript online einreichen". Sie werden automatisch zum Editorial Manager weitergeleitet.

- Bei der ersten Nutzung registrieren Sie sich unter "Register" mit der Angabe einer Benutzer-ID und eines Passwortes. Damit haben Sie bei jeder weiteren Publikation direkten Zugang zum System.

- Nach der Anmeldung finden Sie unter "Author Login" alle notwendigen Hinweise, wie Sie Ihren Beitrag einreichen können.

Sollten Sie noch Fragen zur Manuskripteinreichung haben, wenden Sie sich bitte an:

\section{Wioleta Gorgolik}

Charité Campus Mitte

Schlafmedizinisches Zentrum

Luisenstr.13

10117 Berlin

somnologie@charite.de

www.somnologie.springer.de 